\title{
Simulating Defects in Environmental Sensor Networks Using Stochastic Sensor Models ${ }^{\dagger}$
}

\author{
Sebastian A. Schober ${ }^{1,2, *}$, Cecilia Carbonelli ${ }^{1}$ and Robert Wille ${ }^{2,3}$ \\ 1 Infineon Technologies AG, 85579 Neubiberg, Germany; Cecilia.Carbonelli@infineon.com \\ 2 Institute of Integrated Circuits, Johannes Kepler University Linz, 4040 Linz, Austria; robert.wille@jku.at \\ 3 Software Competence Center Hagenberg GmbH (SCCH), 4232 Hagenberg, Austria \\ * Correspondence: Sebastian.Schober@infineon.com \\ + Presented at the 8th International Symposium on Sensor Science, 17-28 May 2021; Available Online: \\ https://i3s2021dresden.sciforum.net/.
}

check for

updates

Citation: Schober, S.A.; Carbonelli, C.; Wille, R. Simulating Defects in Environmental Sensor Networks Using Stochastic Sensor Models. Eng. Proc. 2021, 6, 88. https://doi.org/ 10.3390/I3S2021Dresden-10094

Academic Editors: Gianaurelio Cuniberti and Larysa Baraban

Published: 17 May 2021

Publisher's Note: MDPI stays neutral with regard to jurisdictional claims in published maps and institutional affiliations.

Copyright: (c) 2021 by the authors. Licensee MDPI, Basel, Switzerland. This article is an open access article distributed under the terms and conditions of the Creative Commons Attribution (CC BY) license (https:// creativecommons.org/licenses/by/ $4.0 /)$.

\begin{abstract}
Chemiresistive gas sensors are an important tool for monitoring air quality in cities and large areas due to their low cost and low power and, hence, the ability to densely distribute them. Unfortunately, such sensor systems are prone to defects and faults over time such as sensitivity loss of the sensing material, less effective heating of the surface due to battery loss, or random output errors in the sensor electronics, which can lead to signal jumps or sensor stopping. Although these defects usually can be compensated, either algorithmically or physically, this requires an accurate screening of the entire sensor system for such defects. In order to properly develop, test, and benchmark corresponding screening algorithms, however, methods for simulating gas sensor networks and their defects are essential. In this work, we propose such a simulation method based on a stochastic sensor model for chemiresistive sensor systems. The proposed method rests on the idea of simulating the defect-causing processes directly on the sensor surface as a stochastic process and is capable of simulating various defects which can occur in low-cost sensor technologies. The work aims to show the scope and principles of the proposed simulator as well as to demonstrate its applicability using exemplary use cases.
\end{abstract}

Keywords: environmental sensors; sensor networks; fault detection

\section{Introduction}

Networks of chemiresistive gas sensors can be used to continuously monitor the different gases in areas of interest with a considerably high spatial density [1]. One major drawback of using this technology can be its stability over long time scales. As different faults can occur over time, the measurement accuracy can degrade consecutively. Such defects can be caused by different processes on the sensor, which can affect the signal output in different ways. Examples would be a loss of sensitivity, sensor stopping, signal jumps, and battery loss, as is shown in Figure 1.

In order to repair or replace individual faulty sensors inside the network, it is necessary to have screening algorithms which continuously evaluate the current state of the sensor network in terms of possible defects. Hence, for general wireless sensor networks, such algorithms have already been investigated [2]. In order to generate and assess such algorithmic approaches for chemiresistive gas sensors, however, simulation data exploring different sensor network scenarios are necessary. For the specific case of sensor drift, such frameworks have already been investigated to evaluate calibration algorithms, e.g., in [3] Therefore, in order to study other fault types, we want to present a framework based upon a stochastic sensor simulator [4], which can provide sensor network simulations specifically feasible for a number of different sensor defects. 


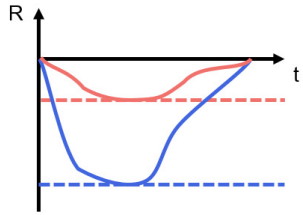

(a)

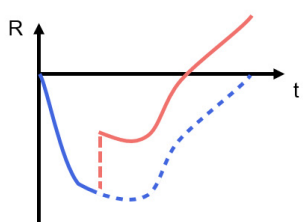

(b)

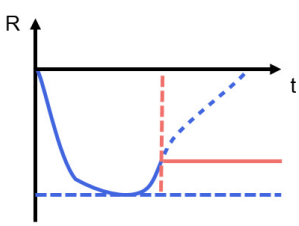

(c)

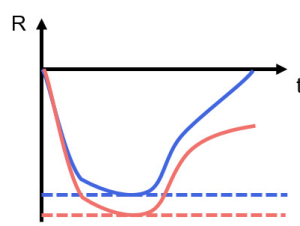

(d)

Figure 1. Different examples of defects (original signal in blue, defect in red). (a) Sensitivity loss leading to a lower resistance response to a gas concentration. (b) Signal jump causing an abrupt additive change in the signal. (c) Sensor stopping leading to constant sensor outputs and (d) battery loss causing a lower heating temperature, which leads to a slower sensor recovery process.

\section{Methods}

Our sensor network simulation framework consists of three different parts, which are depicted in Figure 2a. In the Concentration Model, an array of different gas sources is simulated in order to calculate the spread of the emitted gases across the simulation area and thus simulate their local distributions. The output of this model is the time-dependent concentration series which would be measured at each of the sensor locations. These concentration time series are then the input of the sensor response model. An illustration of the concentration distribution calculated by the concentration model is shown in Figure 2b.

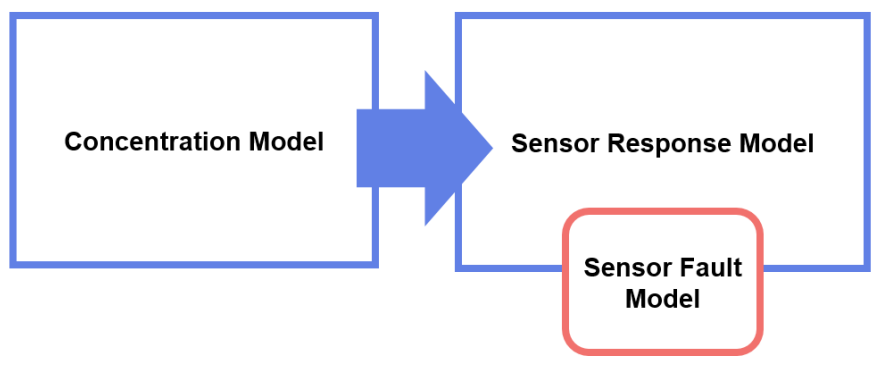

(a)

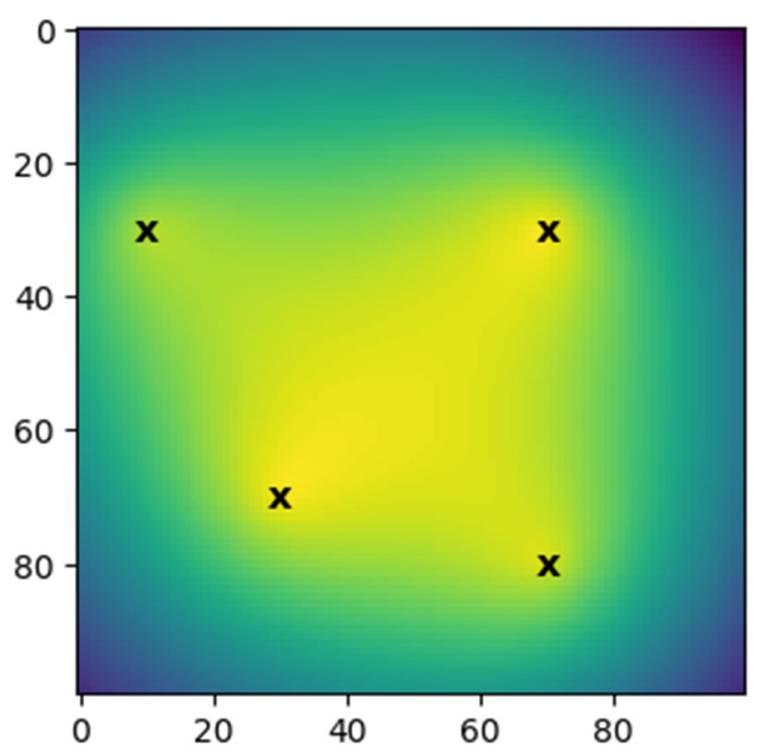

(b)

Figure 2. (a) shows the setup for the simulation framework. (b) shows an exemplary gas distribution created by four gas sources which are marked inside of the image.

The sensor response model then translates the input concentrations at each sensor location to the expected sensor signal measurements. This is done by using a stochastic sensor model which has been developed in previous research [4].

In particular, the sensor response model simulates the processes which are directly occurring on the sensor surface by modeling its adsorption and desorption processes on a microscopic level. Finally, the sensor fault model generates faults in the synthetic signals. Due to the flexibility of the sensor model, two different approaches can be followed here, as shown in Figure 3. 


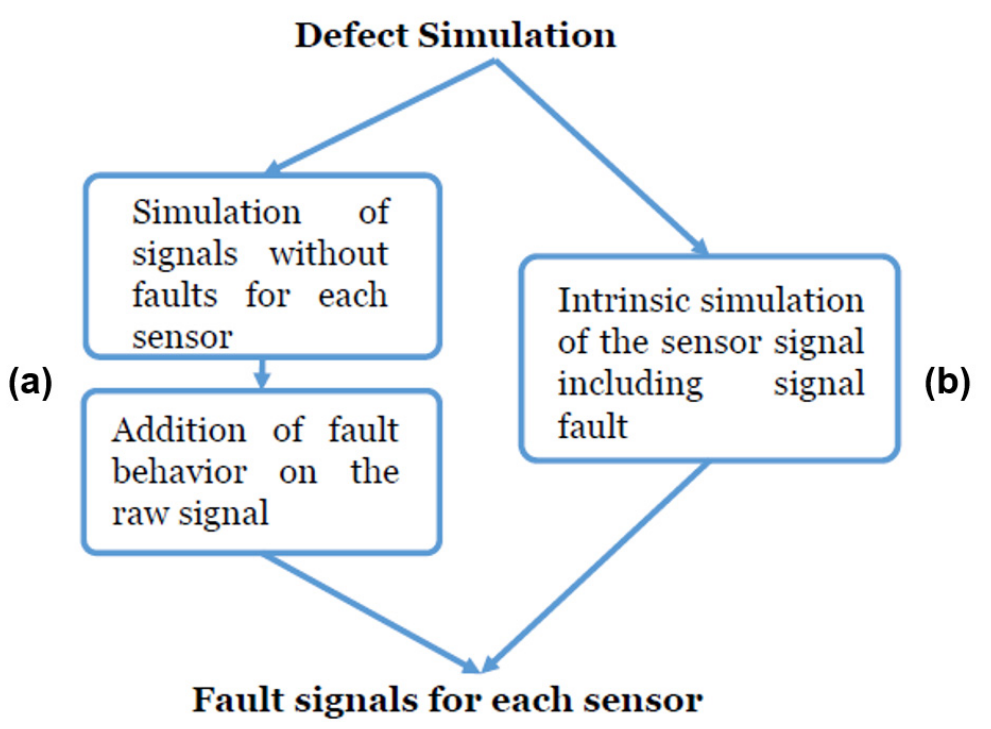

Figure 3. Different approaches for the sensor fault model. (a) shows a post-simulation approach, where the faults are added after the sensor signal was simulated by the sensor response model. (b) shows the intrinsic simulation approach, where the fault behavior is integrated in the sensor response model.

On the one hand, the faults can be generated after the signal simulation by manipulating the output signal (post-simulation faults). On the other hand, the faults can be generated intrinsically in the sensor simulation as well (intrinsic faults). Here, sensitivity loss can be modeled by switching off sensor sites in the sensor surface simulation, whereas battery loss can be generated by changing the heating properties in the model parameters. Both these methods have advantages and disadvantages. Post-simulation faults tend to be rather efficient, since they can be generated spontaneously from a normal signal with respect to the needed sensor fault. However, since a formula is applied here after the simulation, these defects seem to be less accurate. This is the main advantage of the intrinsic approach, since the defect is simulated on the sensor surface, leading to a higher accuracy. This approach is less efficient, however, since every case has to be simulated beforehand.

\section{Results and Discussion}

The different defect types have been implemented intrinsically in the stochastic sensor model by adjusting the heating parameters for battery loss fault simulation and changing the amount of responsive binding sites on the simulation grid for sensitivity loss simulation. Signal jumps and sensor stopping have been implemented as post-simulation faults. In order to test the model, a set of four concentration pulses followed by clean air have been simulated to show the impact of the different faults on the signal. These can be seen in Figure 4.

It can be seen that the sensitivity loss leads to a lower response to the concentration pulses, which is in-line with the physical expectations. Additionally, for the battery loss, which should lead to lower heating capabilities, a signal change can be seen. Here, the recovery process for the sensor signal is not as efficient as for the original signal, which leads to an additive drift caused by slow recovery. Additionally, the processing defects such as sensor stopping and signal jumps are represented well in the simulation experiment. 


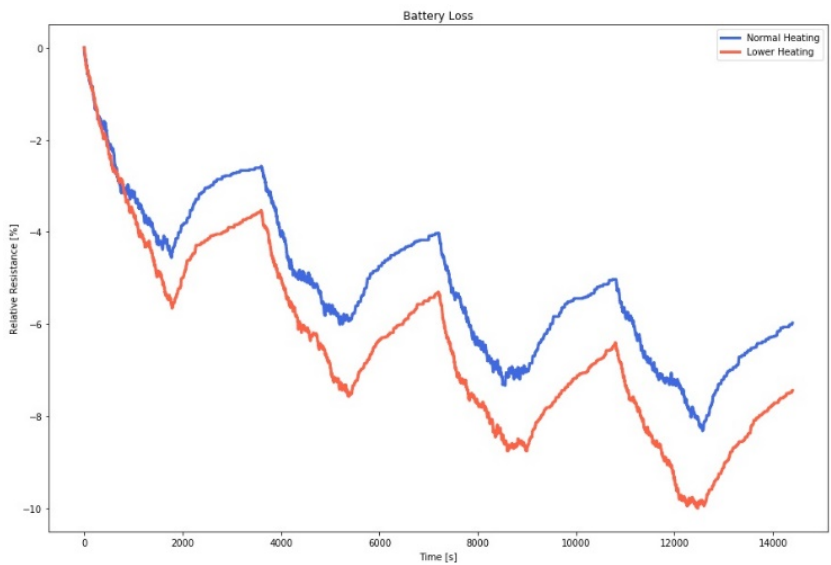

(a)

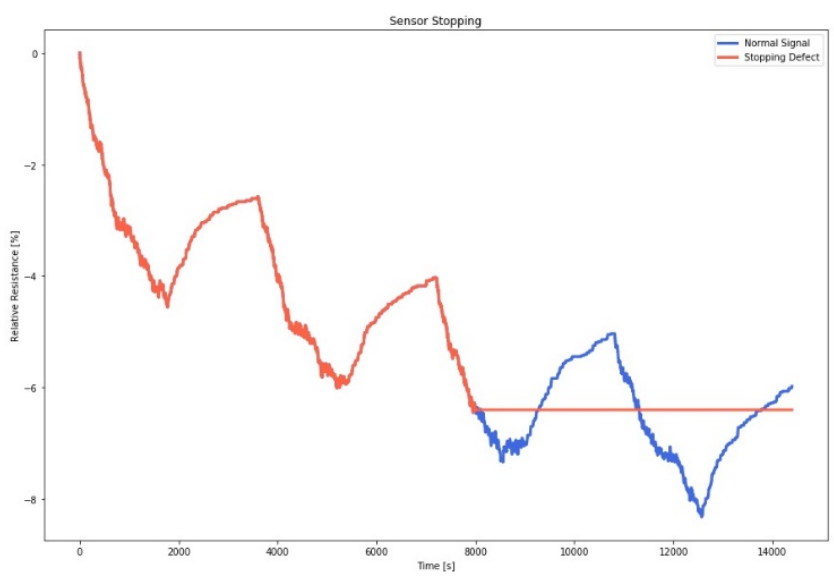

(c)

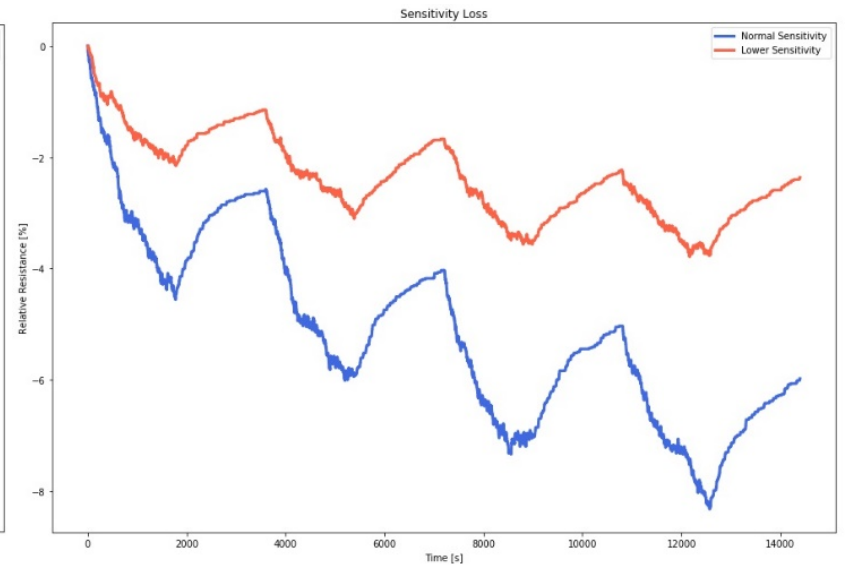

(b)

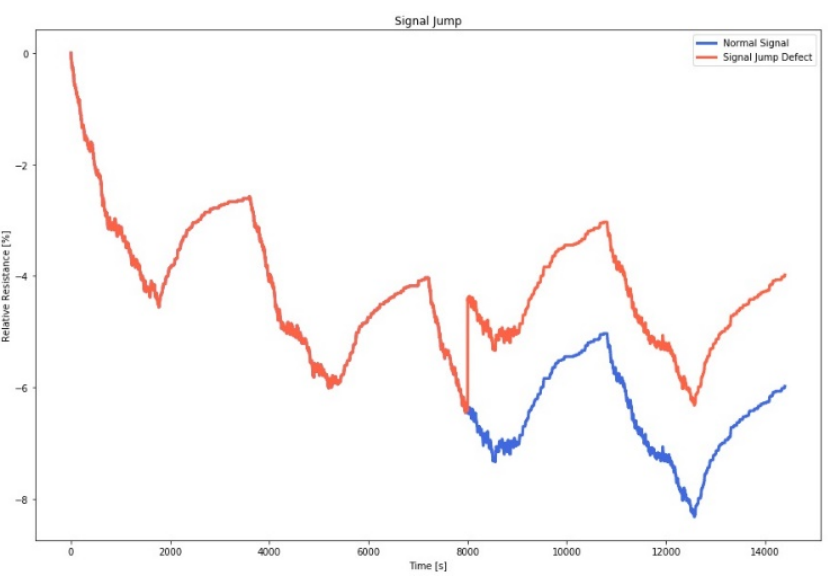

(d)

Figure 4. Different signal outputs for the different sensor fault types implemented in the stochastic model. (a) Sensitivity loss, (b) battery loss, (c) sensor stopping, and (d) signal jumps.

\section{Conclusions and Outlook}

It can be noticed that our simulation framework is suited for simulating various defect effects which can be used for algorithm development for fault detection. There are different design choices to be made which are influenced by computational efficiency and fault accuracy. Therefore, additional research has to be done in this area as well.

In future research, other faults might also be considered for simulation. For example, interfering gases might be an effect which should be studied in more detail for this kind of sensor.

Author Contributions: Conceptualization, S.A.S.; methodology, S.A.S.; software, S.A.S.; validation, S.A.S. and C.C.; formal analysis, S.A.S.; investigation, S.A.S.; resources, S.A.S.; data curation, S.A.S.; writing-original draft preparation, S.A.S. and C.C.; writing-review and editing, S.A.S. and R.W.; visualization, S.A.S.; supervision, C.C. and R.W.; project administration, C.C.; funding acquisition, C.C. All authors have read and agreed to the published version of the manuscript.

Funding: This research received no external funding.

Acknowledgments: This work has been partially supported by the LIT Secure and Correct Systems Lab funded by the State of Upper Austria as well as by the BMK, BMDW, and the State of Upper Austria in the frame of the COMET program (managed by the FFG).

Conflicts of Interest: The authors declare no conflict of interest. 


\section{References}

1. Somov, A.; Baranov, A.; Savkin, A.; Spirjakin, D.; Spirjakin, A.; Passerone, R. Development of wireless sensor network for combustible gas monitoring. Sens. Actuators A Phys. 2011, 171, 398-405. [CrossRef]

2. Mahapatro, A.; Khilar, P.M. Fault Diagnosis in Wireless Sensor Networks: A Survey. IEEE Commun. Surv. Tutor. 2013, 15, 2000-2026. [CrossRef]

3. Delaine, F.; Lebental, B.; Rivano, H. Framework for the Simulation of Sensor Networks Aimed at Evaluating In Situ Calibration Algorithms. Sensors 2020, 20, 4577. [CrossRef] [PubMed]

4. Schober, S.A.; Carbonelli, C.; Roth, A.; Zoepfl, A.; Wille, R. Towards Drift Modeling of Graphene-Based Gas Sensors Using Stochastic Simulation Techniques. In Proceedings of the 2020 IEEE SENSORS, Rotterdam, The Netherlands, 25-28 October 2020; pp. 1-4. [CrossRef] 